\title{
Fiber amplification of pulse bursts at low repetition rates via synchronous pulsed pumping
}

\author{
Hamit Kalaycıŏglu, Seydi Yavaş, F. Ömer Ilday, Koray Eken \\ ${ }^{1}$ Department of Physics, Bilkent University, 06800 Ankara, Turkey \\ ${ }^{2}$ FiberLAST, Ltd., 06531, Ankara, Turkey
}

\begin{abstract}
We report, for the first time, amplification of pulse bursts in Yb-doped fiber at repetition rates as low as $200 \mathrm{~Hz}$ for applications to accelerators and material processing. Synchronous pulsed pumping allows suppression of ASE generation.

(C)2011 Optical Society of America

OCIS codes: (140.0140) Lasers and laser optics; (140.3280) Laser amplifiers
\end{abstract}

There is much interest in fiber amplification of femtosecond and picosecond pulses, which offers certain advantageous features, including low-cost, highly robust and high-gain amplification as well as low added-noise. Previous results have relied on relatively high repetition rate operation (tens of $\mathrm{kHz}$, often $\mathrm{MHz}$ ). While this is the desired regime of operation for most applications, there are a number of niche applications that require lower repetition rates $(<1 \mathrm{kHz})$, which would lead to excessive amplified spontaneous emission (ASE), if pumped continuously. Particularly, applications of fiber laser technology to next-generation accelerator facilities as photoinjector lasers or electron beam diagnostics are promising. These applications [1-3] would require the laser system to exactly match the temporal profile of the electron beam, which typically has the form of a pulse burst containing a few hundred to few thousand pulses with small pulse-to-pulse spacing, as high as $1 \mu \mathrm{s}$, and in some cases less than $1 \mathrm{~ns}$. This pulse burst is then repeated at a low rate, in the $10 \mathrm{~Hz}$ range and up to $1 \mathrm{kHz}$. Material and tissue processing applications can benefit from the ability to generate pulse bursts to better manage thermal effects [4]. In addition, there are niche applications from cavity-enhanced optical parametric chirped-pulse amplification to aeronautics [5], requiring high-repetition rate pulses at high pulse energies, which would correspond to prohibitively high average powers. Burst-mode operation allows a compromise between the requirements of high pulse energy and high repetition rate. Fiber master oscillator power amplifier (MOPA) sources could be ideal front ends for such systems. However, to date, low-repetition-rate burst-mode applications have relied on solid-state laser technology.
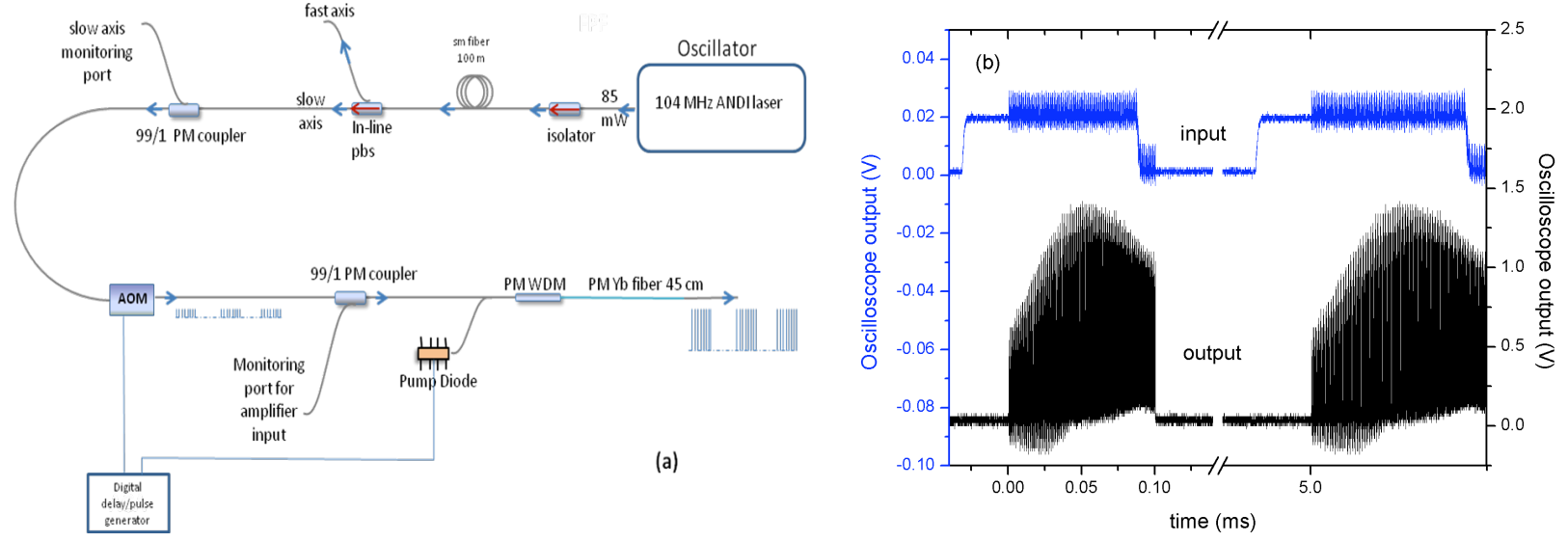

Fig. 1. (a) Schematic diagram of the experimental setup of the Yb-doped amplifier operating in the pulse-burst mode. WMD: wavelength division multiplexer; PBS: polarizing beam splitter; PM: polarization maintaining. (b) Oscilloscope output of input and output pulse train (signal burst $100 \mu$ s, pump pulse $120 \mu$ s, signal lagging pump by $30 \mu$ s, rep. rate $200 \mathrm{~Hz}$ )

Here, we explore, for the first time, to the best of our knowledge, burst-mode operation of Yb-doped fiber amplifier at low repetition rates. For repetition rates lower than approximately $30-50 \mathrm{kHz}$, it is necessary to utilize pulsed pumping in order to avoid ASE generation between the pulse bursts. This operation mode requires detailed understanding of the gain dynamics to minimize ASE generation, while attaining high conversion efficiency. The experimental system (Fig. 1) consists of an all-normal dispersion (ANDi) laser as seed oscillator, a single-mode 
fiber amplifier and synchronized pulse picking and pulsed-pumping electronics. The fiber oscillator operates at a repetition rate of $104 \mathrm{MHz}$ and outputs via a $40 \%$ output coupler an average power of $85 \mathrm{~mW}$ for a $16-\mathrm{nm}$ wide output spectrum centered at $1030 \mathrm{~nm}$. The laser output is polarized with an inline polarization beam splitter, followed by polarization maintaining components. A fiber-coupled acousto-optic modulator (AOM) is used to obtain pulse bursts at desired repetition rates and with desired number of pulses. The pulse bursts are amplified in an $\mathrm{Yb}$-doped fiber amplified pumped by a telecom style fiber-coupled diode with a maximum power of nearly 700 $\mathrm{mW}$, driven in pulsed mode. The AOM and the pump diode are driven from the same digital delay and pulse generator. This ensures that the AOM gate and the pump pulse are synchronized with an adjustable relative delay.

The gain and ASE generation dynamics of the amplifier under pulsed pumping was characterized by investigating the pump-to-signal conversion efficiency, signal gain and amount of ASE formation, as a function of the pump pulse duration and the delay between the signal burst and pump pulse. To begin with, the efficiency of the amplifier was measured in continuous pumping mode with no pulse picking as a benchmark for pulsed performance. In this mode, with $5 \mathrm{~mW}$ of signal power and $400 \mathrm{~mW}$ of pump power, amplified signal power is 200 $\mathrm{mW}$, corresponding to a net gain of $16 \mathrm{~dB}$ and $50 \%$ pump-to-signal conversion.

In the burst mode, the amplifier was operated with signal burst lengths of $20 \mu$ s and $100 \mu$ s at a repetition rate of $1 \mathrm{kHz}$ and with $100 \mu \mathrm{s}$ at $200 \mathrm{~Hz}$. The lower limit of $20 \mu \mathrm{s}$ for the burst mode duration is set by the available signal power. These correspond to pulse burst comprising of 2080 pulses repeated at $1 \mathrm{kHz}$ (duty cycle of $2 \%$ ) and 10800 pulses repeated at $200 \mathrm{~Hz}$ and $1 \mathrm{kHz}$ (duty cycles of $2 \%$ and $10 \%$, respectively). Reliable power measurements could be taken up to a maximum delay of $60 \mu \mathrm{s}$ between pump pulse and signal burst since after this point unabsorbed pump power was observed in the spectrum of the amplifier output. The results of pump-to-signal power conversion measurements are shown in Fig. 2. For each signal burst duration, the conversion efficiency was measured as a function of the delay between rising edges of the pump and signal for different durations of the pump pulse. In the figures, each curve represents a different pump pulse duration denoted by the different duty cycles (pulse duration/pulse repetition rate). Comparing the three cases, the pump conversion efficiencies for $100 \mu$ s are significantly higher as a result of the fact that average signal power is well below saturation for the $20 \mu \mathrm{s}$ case. Also the efficiency improves with increasing duty cycle for $100 \mu$ s going from $200 \mathrm{~Hz}$ to $1 \mathrm{kHz}$ for the same reason. The results for $20 \mu \mathrm{s}$ show that conversion efficiency increases with longer pump pulses and for each pump duration there is an optimum configuration where the falling edge of signal burst comes $10 \mu$ s after the falling edge of the pump pulse. For the 100- $\mu$ s-long burst, the increase in conversion efficiency with the tested pump durations is more gradual with increasing duty cycle of the pump. This is due to the lower ratio of the pump duration to the signal burst length for this case, upper limit of which was dictated by the unabsorbed pump power.
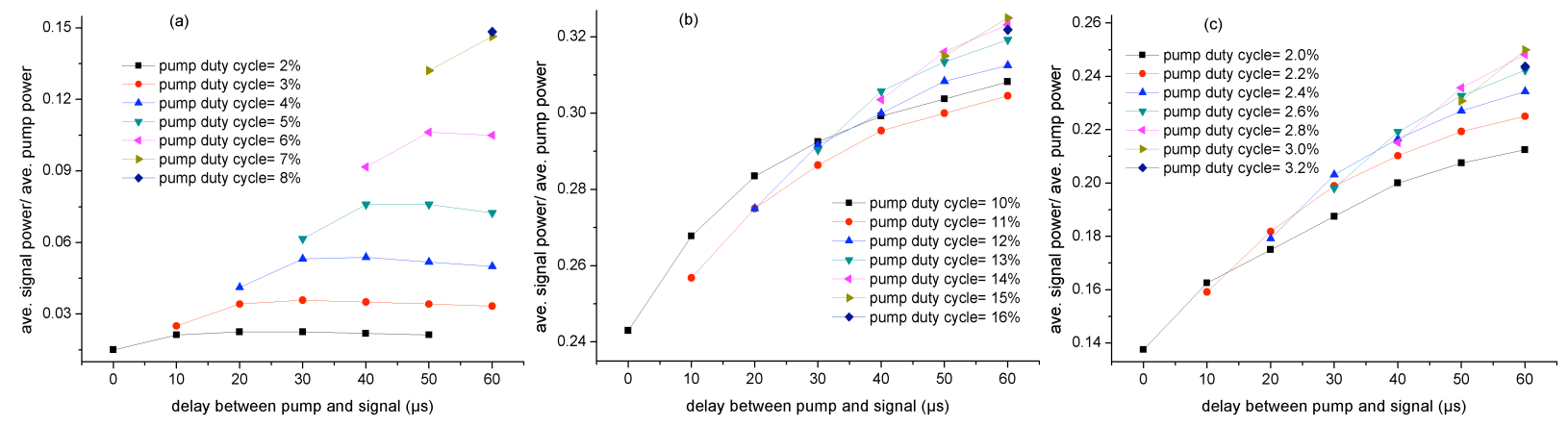

Fig. 2. Pump-to-signal conversion efficiency for pulse burst lengths of (a) $20 \mu \mathrm{s}$, (b) $100 \mu \mathrm{s}$ at $1 \mathrm{kHz}$ and (c) $100 \mu \mathrm{s}$ at $200 \mathrm{~Hz}$ as a function of the delay between the rising edges of the pump and signal emissions.

Fig. 3 (a)-(c) shows the levels of forward ASE produced in the pulse-burst amplifier, for signal bursts of $20 \mu \mathrm{s}$ and $100 \mu \mathrm{s}$ at $1 \mathrm{kHz}$, and $100 \mu \mathrm{s}$ at $200 \mathrm{~Hz}$, respectively. Since the power of ASE relative to the signal provides more relevant information than absolute power, the ratio of signal power to ASE power is given as a function of the delay between pump pulse and signal burst for different pump durations. The signal to ASE ratio is about 30 times higher for $100 \mu \mathrm{s}$ burst duration at $1 \mathrm{kHz}$, and 20 times for $100 \mu \mathrm{s}$ burst duration at $200 \mathrm{~Hz}$, which is expected since the amount of forward ASE predominantly depends on the delay between the rising edges of the pump and signal. Thus, higher signal output levels for the longer signal burst significantly improves this ratio. This characteristic of ASE formation is demonstrated by the degrading signal to ASE ratio with increasing delay between pump and signal clearly observed in each case. The improvement in signal to ASE ratio with increasing pump duration is 
much stronger for the $20 \mu$ s signal burst due to the steeper increase in signal power with pump duration in this case as discussed in the above paragraph.
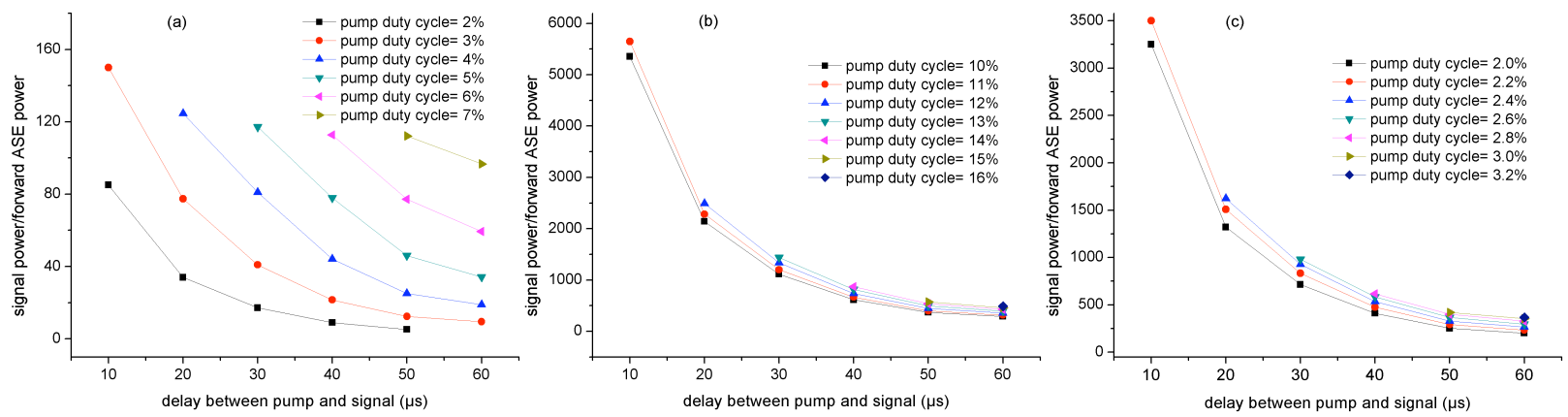

Fig. 3. The measured signal-to-ASE power ratio for pulse burst lengths of (a) $20 \mu \mathrm{s}$, (b) $100 \mu \mathrm{s}$ at $1 \mathrm{kHz}$ and (c) $100 \mu \mathrm{s}$ at $200 \mathrm{~Hz}$ as a function of the delay between the rising edges of the pump and signal emissions.

Amplifier gain for the pulse burst operation was also calculated in terms of the increase in energy per pulse. Results shown in Fig. 4, for $20 \mu \mathrm{s}$ and $100 \mu$ s signal burst durations reveal a trend similar to the pump conversion efficiency. The increase with pump duty cycle in the shorter signal burst case is steeper as the pump pulse duration increase has a bigger impact in this case. The gain is lower than that $(16 \mathrm{~dB})$ of the continuous operation mode in general for all cases and it catches up for the longest pump pulse duration in each case. This indicates that the amplifier is running well below saturation due to low average signal power. The results also show that actual pulse energy gain can exceed the gain for continuous operation by increasing the available pump energy stored per signal pulse by using longer pump pulses.
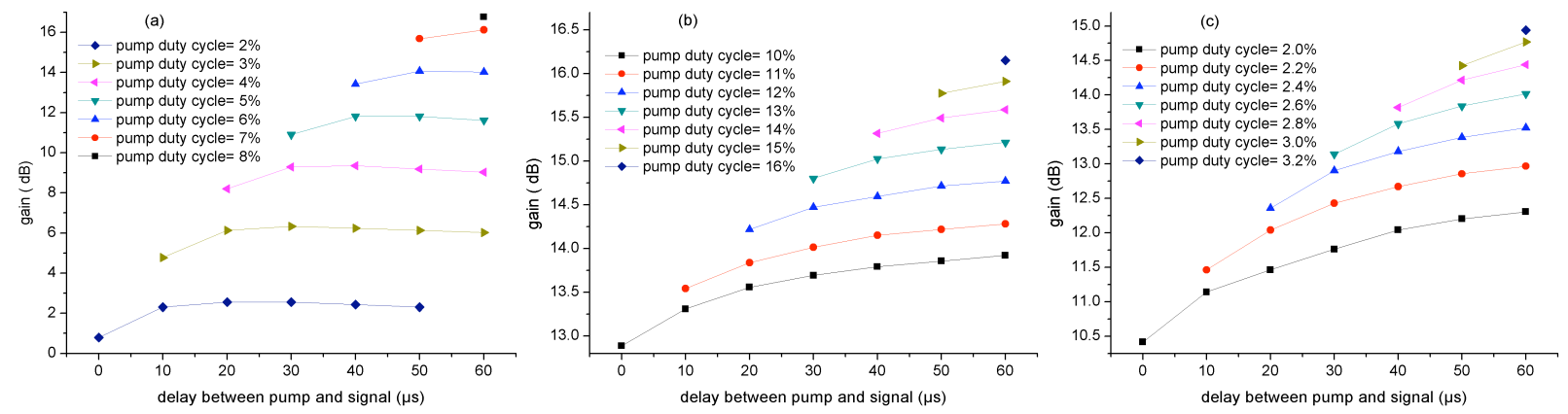

Fig. 4. The measured signal gain factors ratio for pulse burst lengths of (a) $20 \mu \mathrm{s}$, (b) $100 \mu$ s at $1 \mathrm{kHz}$ and (c) $100 \mu$ s at $200 \mathrm{~Hz}$ as a function of the delay between the rising edges of the pump and signal emissions.

In conclusion, we present initial results on the amplification of low-repetition-rate pulse bursts via synchronous pulsed pumping of an Yb-doped fiber amplifier. Amplification at repetition rates as low as $200 \mathrm{~Hz}$ has been accomplished while suppressing ASE to better than $30 \mathrm{~dB}$, which would not be possible without the use of pulsed pumping. Practically, this approach allows the generation of the virtually any pulse pattern, as long as the desired adjacent pulse-to-pulse separation matches the repetition rate of the oscillator or an integer fraction of it. As a next step, multiple stages of amplification with stronger pump lasers will be cascaded to reach higher pulse energies.

\section{References}

[1] H. Braun, et al., "CLIC 2008 Parameters," CERN-OPEN-2008-021, 2008.

[2] M. Altarelli, et al., "The European X-Ray Free-Electron Laser Technical Design Report,” http://www.xfel.net, 2007.

[3] F. Ö. Ilday, A. Winter, and F. X. Kärtner, "Pulse shaping for a long-distance optical synchronization system", IEICE Trans. Electron. E90-C, 450 (2007).

[4] M. Murakami, B. Liu, Z. Hu, Z. Liu, Y. Uehara, and Y. Che, "Burst-mode femtosecond pulsed laser deposition for control of thin film morphology and material Ablation," Appl. Phys. Expr. 2, 042501 (2009).

[5] P. Wu, W. R. Lempert, and R. B. Miles, "Megahertz pulse-burst laser and visualization of shock-wave/boundary-layer interaction," AIAA J. 38, 672 (2000). 